\title{
Analysis of Open Stub Resonator and its Application in Dual Isolation Band of SPDT Switch Design
}

\author{
M. Y. Algumaei, N. A. Shairi, Z. Zakaria, A. M. S. Zobilah, B. H. Ahmad \\ Center for Telecommunication Research \& Innovation (CeTRI), Faculty of Electronics and Computer Engineering, \\ Universiti Teknikal Malaysia Melaka (UTeM), Hang Tuah Jaya, 76100, Durian Tunggal, Melaka, Malaysia
}

\begin{tabular}{l} 
Article Info \\
\hline Article history: \\
Received Jun 9, 2016 \\
Revised Sep 15, 2016 \\
Accepted Sep 29, 2016
\end{tabular}

Keyword:

Bandstop to allpass Open stub resonator RF/Microwave switch SPDT switch

Transmission line stub

\begin{abstract}
In this paper, an analysis of open stub resonator is presented and its application in dual isolation band of Single Pole Double Throw (SPDT) switch is proposed. A mathematical model and the characteristic of the bandstop of the resonator were analyzed and discussed. The open stub resonator was implemented using the microstrip transmission line and able to switch between bandstop and allpass responses. Frequency bands of 2.3 and $3.5 \mathrm{GHz}$ were chosen to demonstrate the dual isolation band in the switch design. The performance results of the SPDT switch showed that the isolation was greater than $30 \mathrm{~dB}$, return loss was greater than $10 \mathrm{~dB}$ and insertion loss less than $2 \mathrm{~dB}$ at the center resonant frequency of 2.3 and $3.5 \mathrm{GHz}$. The potential application of the proposed dual isolation band of SPDT switch is for multi band RF front-end system such as WiMAX, LTE, WiFi and HyperLAN.
\end{abstract}

Copyright $\odot 2016$ Institute of Advanced Engineering and Science. All rights reserved.

\section{Corresponding Author:}

Noor Azwan Shairi,

Faculty of Electronics and Computer Engineering,

Universiti Teknikal Malaysia Melaka (UTeM),

Hang Tuah Jaya-76100, Durian Tunggal, Melaka, Malaysia.

Email: noorazwan@utem.edu.my

\section{INTRODUCTION}

In general, resonators in RF/microwave can be used in many applications such as antennas [1-3] filters [4-5], switches [6-7], amplifier [8], absorber [9] and others. These resonators are generally realized using microstrip technology where they can be implemented in different shape and size such as transmission line [10], radial [8], ring [3], [9], metamaterial [11] and coupled line [12]. As described in [13], the operation of RF/microwave resonators is very similar to that of the lumped-element resonators of circuit theory, whereby it can be modelled either as a series or parallel resistor, inductor and capacitor $(R L C)$ resonant circuit. On the other hand, by open-circuited at one end of a stub resonator (known as an open stub resonator), it resonates at a certain frequency with specific electrical length [13-14].

In the last few years, researches on the switchable, tunable and reconfigurable of $\mathrm{RF} /$ microwave devices are kept increasing due to the demand on the multi band and multi standard in wireless communication system (e.g. WiFi, WiMAX, LTE, WiBro, HiperLAN and etc.) [15-20]. The main reason is researchers and engineers have to design a RF front-end with less expensive and smaller size than simply putting multiple RF/microwave components next to each other. Hence, from literatures, there are several techniques and solutions on the switchable, tunable and reconfigurable in RF/microwave devices as reported in [19], [21-24].

Meanwhile, in designing RF switches, there are the demand on the wide-, broad- or multi-band RF switches as reported in [25]. Figure 1 shows an example of multi band RF front-end system where Single 
Pole Double Throw (SPDT) switch is used to support two different frequency bands. Thus, dual band isolation is needed to support the multi band RF front-end system. As discussed by the authors in [25], RF switch is one of the solution to reduce the size of wide-, broad- or multi-band RF front-end system. The integration of multiple wireless systems is required either for different standards (e.g. WiFi and WiMAX) or different spectrum allocations at various locations (e.g. different WiMAX spectrums in different countries).

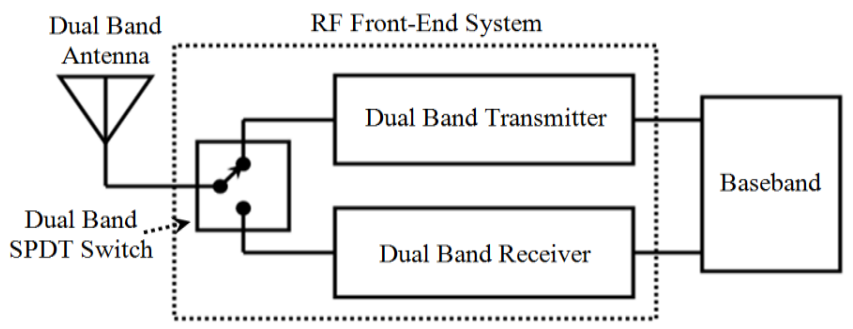

Figure 1. General application of SPDT switch in multi band RF front-end system

Therefore, this paper analyzes an open stub resonator and proposes its application for a dual isolation band of SPDT switch. In the proposed SPDT circuit, the open stub resonator was implemented using the microstrip transmission line and could be switched between bandstop and allpass responses. Frequency bands of 2.3 and $3.5 \mathrm{GHz}$ were chosen to demonstrate the dual isolation band in the switch design. It could be used in any multi band RF front-end system such as WiMAX, LTE, WiFi and HyperLAN.

This paper is organized as follows. Section 2 discusses a theory of open stub resonator based on the mathematical model and a realization of the resonator using the microstrip transmission line. A switchable open stub resonator is described in this section as well. Meanwhile, Section 3 presents the application of the open stub stub resonator in dual isolation band of SPDT switch. The circuit configuration and operation are described in this section. Then, the results and analyses of the modeled open stub resonator and the performance of the proposed SPDT switch are described in Section 4. Finally, the work is concluded in Section 5 .

\section{OPEN STUB RESONATOR}

\subsection{General Theory of Open Stub Resonator}

Figure 2 is a general diagram of open stub resonator, whereby it was open-circuited at one end of the stub resonator and connected in shunt with $50 \Omega$ transmission line. The open stub resonator could be realized using transmission line stub.

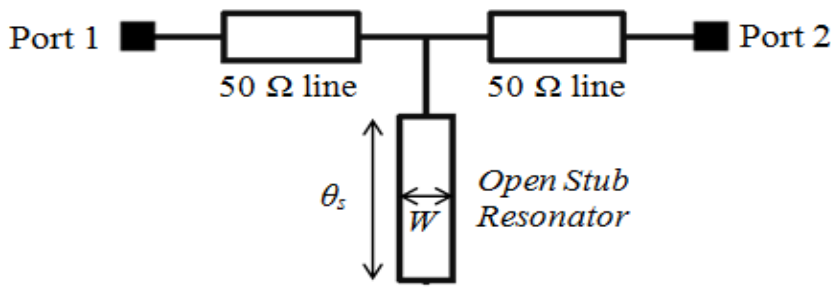

Figure 2. General diagram of open stub resonator

The open stub resonator was mathematically modeled whereby the input impedance of the resonator [13] was written as

$$
Z_{\text {in }}=-j Z_{s} \cot \theta=\frac{Z_{s}}{j \tan \theta_{s}}
$$

where $Z_{s}$ is characteristic impedance of open stub resonator and $\theta_{s}$ is electrical length in degree. From (1), the $A B C D$ matrix of the open stub resonator was given by 
$\left[T_{S}\right]=\left[\begin{array}{cc}1 & 0 \\ Y_{\text {in }} & 1\end{array}\right]=\left[\begin{array}{cc}1 & 0 \\ \left(\frac{j \tan \theta_{S}}{Z_{S}}\right) & 1\end{array}\right]$

Then, the $S_{12}$ and the $S_{21}$ of the open stub resonator were obtained by converting the $A B C D$ matrix in (2) to $S$-parameter. Hence,

$$
\begin{aligned}
S_{12} & =S_{21}=\frac{2}{A+\frac{B}{Z_{0}}+C Z_{0}+D} \\
& =\frac{2}{1+\frac{0}{Z_{0}}+\left(\frac{j \tan \theta_{s}}{Z_{S}}\right) Z_{0}+1} \\
& =\frac{2}{2+\left(\frac{j \tan \theta_{S}}{Z_{S}}\right) z_{0}} .
\end{aligned}
$$

For a simple analysis and discussion of attenuation response of stub resonators, consider a normalized characteristic of impedance where $Z_{0}=1$ and impedance of resonator, $Z_{s}=Z_{0}=1$. In order to produce attenuation or notch response of the open stub resonator, the electrical length was $\theta_{s}=90^{\circ}$ (or $\pi / 2$ radian). Hence, the $S$-parameter of (3) becomes,

$$
S_{12}=S_{21}=\frac{2}{2+\left(\frac{j \tan \left(\frac{\pi}{2}\right)}{1}\right)} \approx 0
$$

or in decibel

$$
\left|S_{12}\right|^{2} \mathrm{~dB}=\left|S_{21}\right|^{2} \mathrm{~dB}=20 \log _{10}(0)=\infty \mathrm{dB} .
$$

From (5), an ideal infinite attenuation or notch was obtained when the electrical length of the open stub resonator was a quarter wave $(\lambda / 4)$. In degree and radian, they were $90^{\circ}$ and $\pi / 2$ radian respectively. These attenuation characteristics were used to produce high isolation of SPDT switch. The next sections analyze in detail $S_{21}$ (attenuation or notch response) for the open stub resonator using transmission line.

\subsection{Transmission Line as an Open Stub Resonator}

Transmission line is a simple stucture that can be designed as an open stub resonator. It was realized by using microstrip line based on FR4 substrate. Therefore, to analyze the attenuation or notch $\left(S_{21}\right)$ of transmission line stub resonator, the impedance of the resonator, $Z_{s}$ was replaced with mathematical model of characteristic impedance of microstrip line [13], hence

$$
Z_{s}=\frac{120 \pi}{\sqrt{\epsilon_{e}}\left[\frac{W}{d}+1.393+0.667 \ln \left(\frac{W}{d}+1.444\right)\right]}
$$

where $\epsilon_{e}$ is effective dielectric constant, $d=1.6 \mathrm{~mm}$ for FR4 substrate thickness, and $W=2.9 \mathrm{~mm}$ for width of microstrip line in the range between 2 and $4 \mathrm{GHz}$.

Then, (3) was rearranged with (6), and the $S_{21}$ was given by

$$
S_{21}=\frac{2}{2+\frac{j Z_{0} \tan \left(\theta_{S}\right) \sqrt{\epsilon_{e}}\left(\frac{W}{d}+1.393+0.667 \ln \left(\frac{W}{d}+1.444\right)\right)}{120 \pi}} .
$$

To calculate the physical length (in meter) of $\lambda / 4$ length of transmission line stub resonator [13],

$$
\theta_{s}=\beta l=\sqrt{\epsilon_{e}} k_{0} l
$$

where

$$
k_{0}=\frac{2 \pi f}{c} .
$$


Therefore, (8) was rearranged and was substituted by $=\frac{\pi}{2}$, while the $\lambda / 4$ length of the transmission line stub resonator was determined as

$$
l=\frac{\frac{\pi}{2}}{\sqrt{\epsilon_{e}}\left(\frac{2 \pi f}{c}\right)} \text { meter }
$$

where $c$ is speed of light and $f$ is resonant frequency. Based on these derivations, they were used to determine the suitable length and width of transmission line stub resonator that had been associated with resonant frequency and attenuation or notch $\left(S_{21}\right)$ of the resonator.

\subsection{Switchable Open Stub Resonator}

A PIN diode was connected in between the microstrip line and open stub resonator (in tranmission line stub resonator), hence a switchable open stub resonator could be performed by giving a different biasing voltage. The resonator that was connected to a microstrip line is illustrated in Figure 3(a). As depicted in Figure 3(b), if a positive voltage was applied $(+5 \mathrm{~V})$, the PIN diode, $D$ would be in the ON state and the transmission line stub resonator would be connected to the microstrip line. In this condition, it operated as a bandstop filter due to the quarter wave $(\lambda / 4)$ line of the open stub resonator, converting from an open to short circuit to the main microstrip transmission line. If a negative voltage $(-5 \mathrm{~V})$ was applied in Figure $3(\mathrm{c}), D$ would be in the OFF state and the transmission line stub resonator would be disconnected from the microstrip line. In this condition, the transmission line stub resonator responded as an allpass between Port 2 and Port 1.

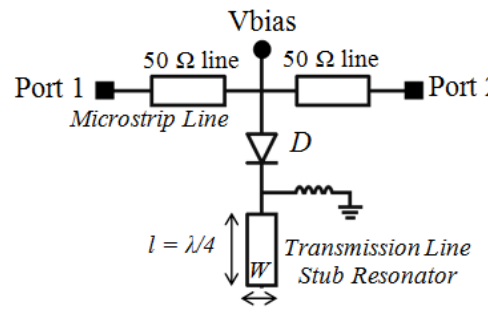

(a)

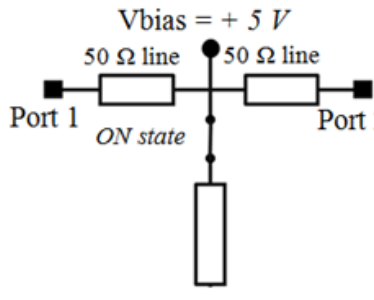

(b)

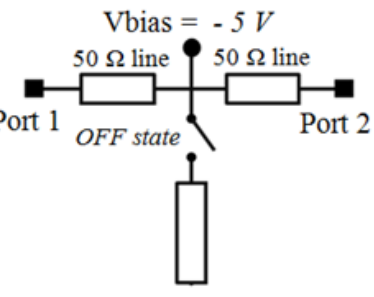

(c)

Figure 3. Circuit Diagram of Switchable Transmission Line Stub Resonator, (a) Circuit Operation: (b) ON State (Bandstop Response) and (c) OFF State (Allpass Response)

The actual responses of the switchable resonators without and with PIN diode need to be analyzed and discussed. This is important because any switching element such as MEMs and PIN diodes can deteriorate circuit's performances as reported in [1], [26]. It is due to the parasitic reactance (e.g. inductance or capacitance) or resistance in the switching elements. If possible, compensation must be carried out in the simulation stage on the switchable resonator design. For instance, reducing length of a microstrip resonator is one of the techniques used to compensate the parasitic reactance that affects the filter's performance [26].

\section{OPEN STUB RESONATOR IN DUAL ISOLATION BAND OF SPDT SWITCH}

As shown in Figure 4, dual isolation band of SPDT switch with open stub resonators (based on microstrip transmission line) was designed in $2.3 \mathrm{GHz}$ and $3.5 \mathrm{GHz}$. The switchable open stub resonators of $S 1$ and $S 2$ were cascaded with series PIN diode of D5 in transmit arm, while the resonators of $S 3$ and $S 4$ were cascaded with series PIN diode of D6 in receive arm. The $S 2$ and $S 3$ are resonated at $2.3 \mathrm{GHz}$ while the $S 1$ and $S 4$ are resonated at $3.5 \mathrm{GHz}$. In this design, the isolation of the SPDT is depend on these open stub resonators due to its attenuation (or notch) performance and also the series PIN diodes.

Besides, quarter wavelength lines $(\lambda / 4)$ had been used in the circuit of transmit and receive arms where they were placed between the resonators and the series PIN diode. The purpose of the $\lambda / 4$ lines is to transform from low impedance of transmission line stub resonator to high impedance in the microstrip line. So, this would prevent any RF leakage between transmit (Port 1) and receive (Port 3) arms where it is measured as isolation, $S_{31}$. 


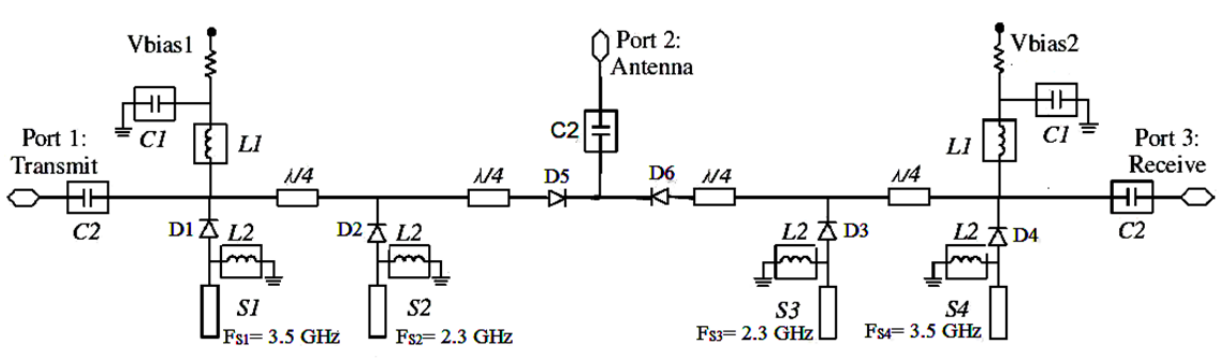

Figure 4. Circuit Diagram of the Proposed Dual Isolation Band of SPDT switch with Switchable Open Stub Resonators

Figure 5 shows the circuit operation of the proposed dual isolation band of SPDT switch. Due to the symmetrically construction of the SPDT circuit, for the circuit operation, the transmit mode is only discussed in this paper. Hence, in transmit mode, PIN diodes of D1 and D2 were in OFF state with voltage control of $+5 \mathrm{~V}$, while PIN diodes of D3 and D4 were in ON state with voltage control of $-5 \mathrm{~V}$. For the series PIN diodes, the D5 was in ON state while the D6 was in OFF state. In this case, both $S 1$ and $S 2$ created an allpass response and the D5 was in short circuited. Thus, RF signals propagated from transmit (Port 1) to antenna (Port 2) with low insertion loss. On the other hand, the switchable open stub resonators of $S 3$ and $S 4$ in the receive arm performed as a bandstop filter and the D6 was in open circuited. As consequent, these conditions created high isolation at 2.3 and $3.5 \mathrm{GHz}$ in the SPDT switch between transmit and receive ports.

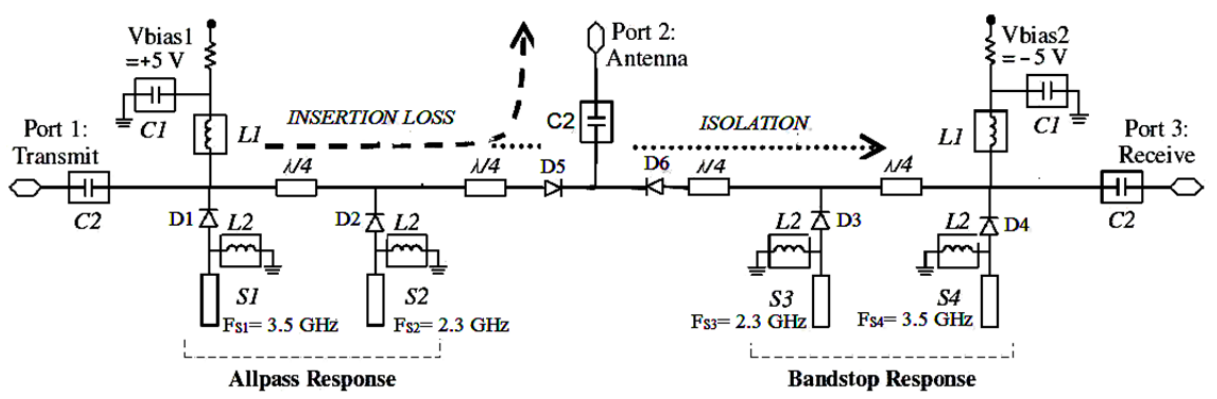

Figure 5. Circuit Diagram of Dual Isolation Band of SPDT switch with Switchable Open Stub Resonators

Table 1 presents a summary of the circuit operation during receive and transmit modes of the proposed SPDT switch. Meanwhile, the design of the dual isolation band of SPDT switch circuit in Figure 5, was constructed in Advanced Design System (ADS) software for $S$-parameter performance simulation and layout design. To build up the circuit, microstrip model in ADS was used by considering the FR4 substrate parameters such as board thickness $=1.6 \mathrm{~mm}$ and relative dielectric constant, $\varepsilon_{r}=4.7$.

Table 1. Summary of Circuit Operation of the Proposed SPDT switch with Switchable Open Stub Resonators

\begin{tabular}{lll}
\hline & Transmit Mode & Receive Mode \\
\hline Vbias1 & +5 Volt & -5 Volt \\
Vbias2 & -5 Volt & +5 Volt \\
Series PIN diode (D5) & ON state & OFF state \\
Series PIN diode (D6) & OFF state & ON state \\
Shunt PIN diodes (D1 \& D2) & OFF state & ON state \\
Shunt PIN diodes (D3 \& D4) & ON state & OFF state \\
Open Stub Resonators (S1 \& S2) & Allpass response & Bandstop response \\
Open Stub Resonators $(S 3 \& S 4)$ & Bandstop response & Allpass response \\
\hline
\end{tabular}

The parasitic elements of PIN diodes such as junction capacitance, $C_{j}$ and series inductance, $L_{s}$ were considered in the resonator design and through the entire simulation processes. Hence, the final dimensions 
of the open stub resonators were $W_{l}=5 \mathrm{~mm}$ and $l_{l}=11.3 \mathrm{~mm}$ at $2.3 \mathrm{GHz}$ band and $W_{2}=2.9 \mathrm{~mm}$ and $l_{2}=7.15 \mathrm{~mm}$ at $3.5 \mathrm{GHz}$ band. The final layout of the proposed SPDT switch with switchable open stub resonators is illustrated in Figure 6.

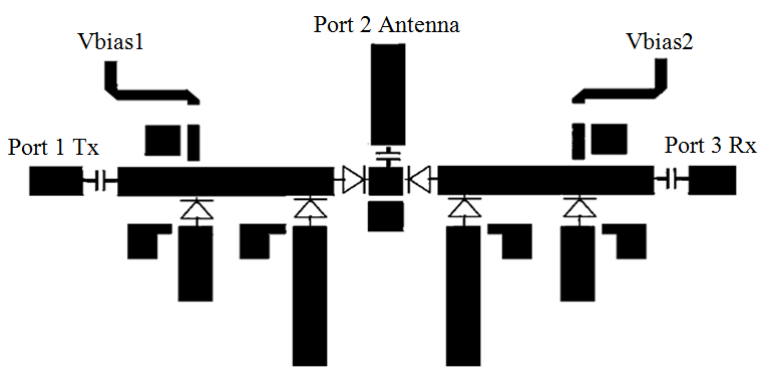

Figure 6. Layout of the Proposed SPDT switch with Switchable Open Stub Resonators

\section{RESULTS AND DISCUSSIONS}

\subsection{Open Stub Resonator}

Figure 7 shows the characteristics of open stub resonator based on microstrip transmission line for impedance versus width (Figure 7(a)), and attenuation pole versus width (Figure 7(b)). The graph in Figure 7(a) was calculated using (6), while the graph in Figure 7(b) was calculated using (7). The calculated values were based on the different values of width of transmission line stub selected from 1 to $10 \mathrm{~mm}$. The calculated transmission line stub impedance, Zs, and attenuation pole were based on FR4 substrate with thickness of $1.6 \mathrm{~mm}$ and dielectric constant, $\epsilon_{\mathrm{r}}$ of 4.7 .

It was observed that the graph in Figure 7(a) shows a negative exponential curve, whereby the impedance of transmission line stub resonator was varied by changing the width of the resonator, $W$. Then, the graph in Figure7(b) shows the different values of attenuation pole of transmission line stub resonator for different widths simply due to the varying values of impedance, $Z_{s}$ (also a function of $W$ ). These attenuation characteristics were used to produce dual isolation band in SPDT switch design.

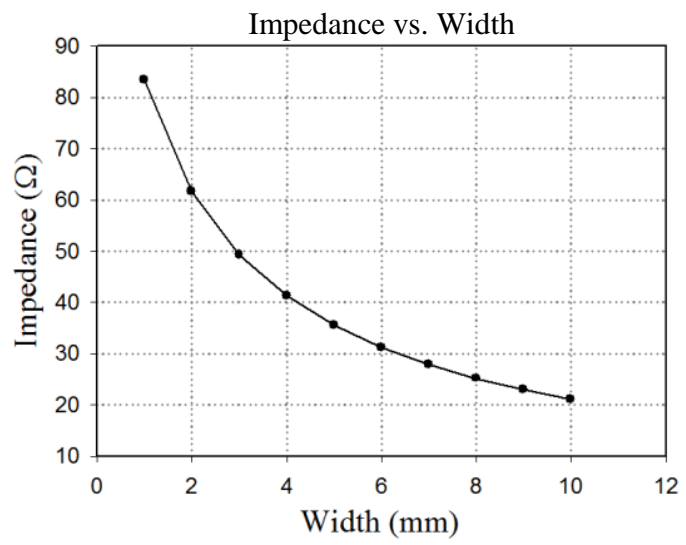

(a)

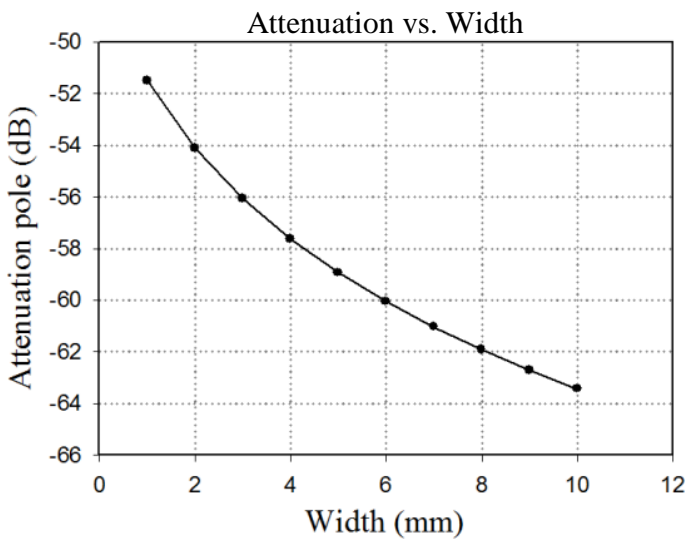

(b)

Figure 7. Characteristics of Transmission Line Stub Resonator for (a) Impedance Versus Width, and (b) Attenuation Pole Versus Width

Since the bandstop response of the switchable open stub resonator was operated during the ON state of the PIN diode, Figures 8(a) and (b) show a deterioration of the simulated bandstop response when the PIN diode (BAP64-02 model) had been incorporated with the transmission line stub resonator (MLIN model) in the ADS software. The original transmission line stub resonator without PIN diode was designed according to the mathematical analysis in Figure 7 and (9). Thus, the selected values of the width and length of the stub resonator were $W=2.9 \mathrm{~mm}$ and $l=11.3 \mathrm{~mm}$ (resonated at $3.5 \mathrm{GHz}$ ). 
As a comparison between open stub resonator without and with PIN diode, there was a frequency shift when the transmission line stub resonator had been attached with a PIN diode. As shown in Figure 9(a) it shifted from $3.5 \mathrm{GHz}$ to $2.86 \mathrm{GHz}$, where there was $18.3 \%$ of frequency shift from $3.5 \mathrm{GHz}$. The frequency shift was due to the changes of reactance of the original transmission line stub resonator by the parasitic inductance of the PIN diode (ON state). It increased the total inductance in the circuit, hence the resonant frequency shifted to the lower frequencies. Besides, it was found that the attenuation had also dropped from $31.5 \mathrm{~dB}$ to $23.8 \mathrm{~dB}$, giving $24.4 \%$ of attenuation reduction from $31.5 \mathrm{~dB}$ attenuation. The attenuation reduction was due to the inductance, $L_{i}$ and resistance, $R_{f}$ of the PIN diodes. Figure 8 (b) shows a change of return loss response between transmission line stub resonator with and without PIN diode.

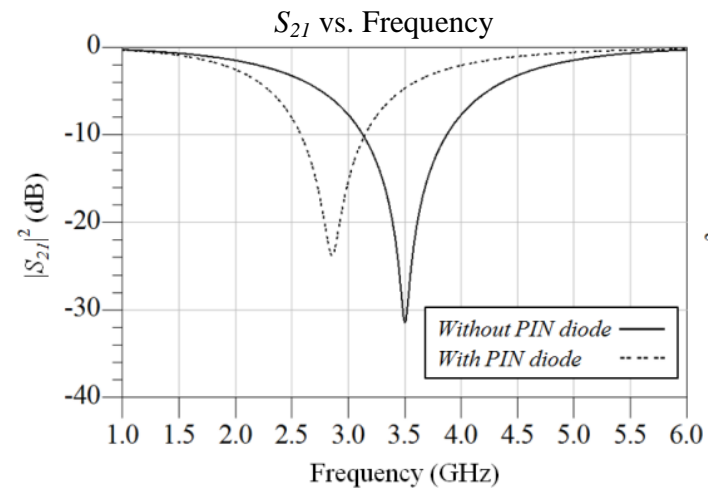

(a)

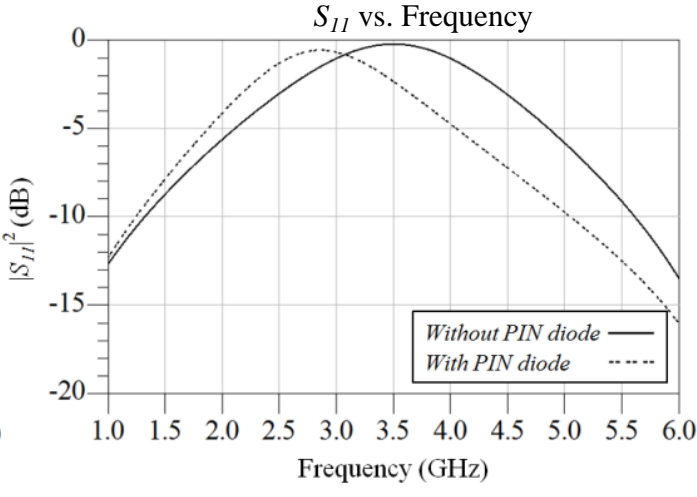

(b)

Figure 8. Bandstop Response of Transmission Line Stub Resonator with and without PIN Diode; (a) $S_{21}$ and (b) $S_{11}$

Figure 9 generally shows a frequency responses between bandstop and allpass of the switchable transmission line stub resonator. Comparison was made between without and with PIN diode (after optimization). The $S_{21}$ graph was plotted from 1 to $6 \mathrm{GHz}$. Figures 9(a) is the bandstop response while Figures 9(b) is the allpass response. As a solution to the shift of resonant frequency, the length of the original transmission line stub resonator had to be readjusted in order to compensate the frequency shift due to the parasitic inductance of PIN diode (ON state). By reducing the stub resonator length to $8.65 \mathrm{~mm}$, the resonant frequency had shifted back to $3.5 \mathrm{GHz}$. It was $23 \%$ of length reduction from the original length, $11.3 \mathrm{~mm}$. Besides, the simulated attenuation was $23.6 \mathrm{~dB}$, which was $7.9 \mathrm{~dB}$ lower than the attenuation of the original stub resonator, $31.5 \mathrm{~dB}$.

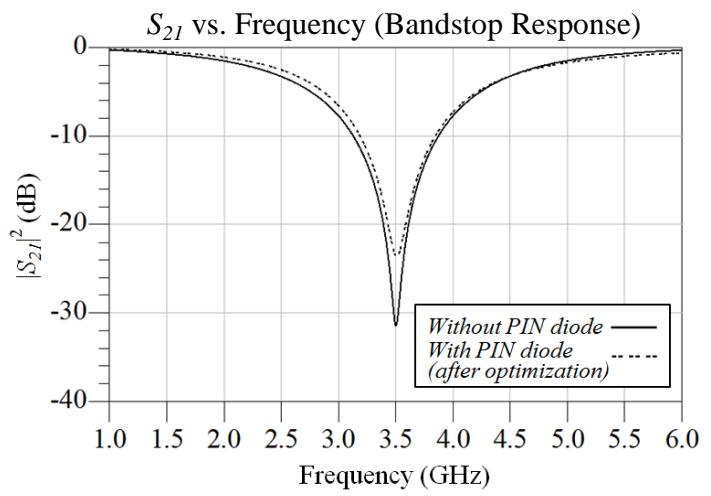

(a)

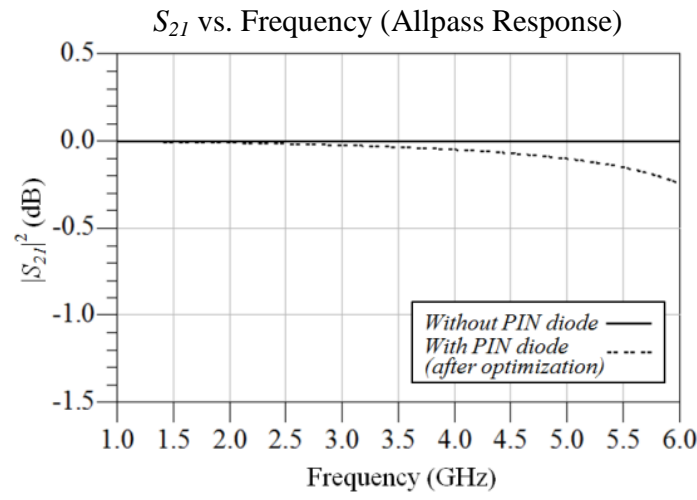

(b)

Figure 9. Frequency Responses of Switchable Transmission Line Stub Resonator (After Optimization), Bandstop Response (a) $S_{21}$ and (b) $S_{11}$, Allpass Response (c) $S_{2 l}$ and (d) $S_{11}$ 


\subsection{Dual Isolation Band of SPDT Switch}

Figures 10 shows the performance results of the proposed SPDT switch in $2.3 \mathrm{GHz}$ and $3.5 \mathrm{GHz}$ bands. As shown in Figures 10(a), for both operation frequencies $(2.3 \mathrm{GHz}$ and $3.5 \mathrm{GHz})$, the isolation performance, S31 of the SPDT switch was more than $30 \mathrm{~dB}$. It shows that at $2.3 \mathrm{GHz}(\mathrm{m} 1)$, the simulated isolation was $46.78 \mathrm{~dB}$ while at $3.5 \mathrm{GHz}(\mathrm{m} 2)$, the simulated isolation was $44.74 \mathrm{~dB}$. It can be noted that, the high isolation was due to the combination of the bandstop (attenuation or notch) response of the open stub resonator and the open circuited of the series PIN diode.

Meanwhile, Figure 7(b) shows the simulated result of return loss, $\mathrm{S} 11$ in $2.3 \mathrm{GHz}$ and $3.5 \mathrm{GHz}$ band, where at $2.3 \mathrm{GHz}(\mathrm{m} 3)$, the return loss was $20.83 \mathrm{~dB}$ and at $3.5 \mathrm{GHz}(\mathrm{m} 4)$ the return loss was 15.16 $\mathrm{dB}$. Figure 7(c) shows the simulated insertion loss, $\mathrm{S} 21$ where at $2.3 \mathrm{GHz}(\mathrm{m} 5)$, the $\mathrm{S} 21$ was $0.359 \mathrm{~dB}$ and at $3.5 \mathrm{GHz}(\mathrm{m} 6)$, the $\mathrm{S} 21$ was $0.623 \mathrm{~dB}$. Hence, it can be concluded that the return loss and insertion loss are very good performance where the return loss must be less than $10 \mathrm{~dB}$ and the insertion loss normaly must be less than $2 \mathrm{~dB}$.

The performance of the proposed SPDT switch is summarized in Table 2. It summarizes the performance in terms of isolation, return loss and insertion loss at 2.3 and $3.5 \mathrm{GHz}$.

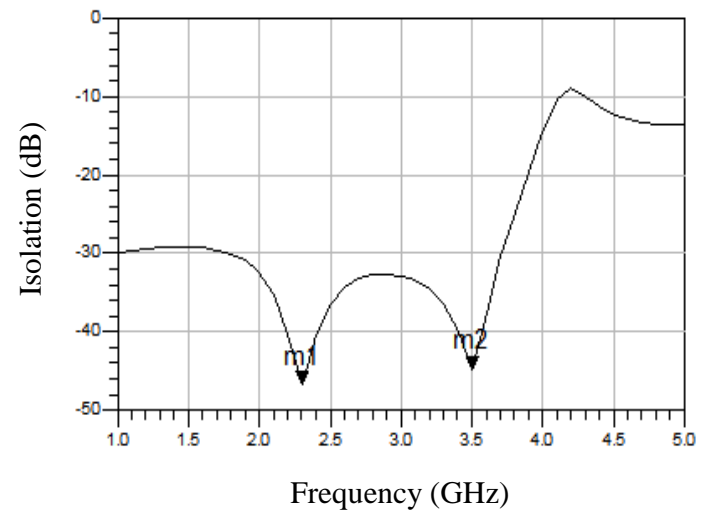

(a)

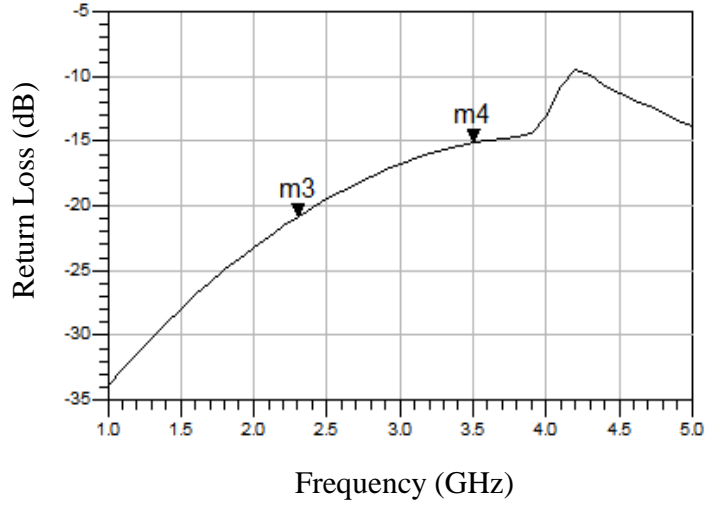

(b)

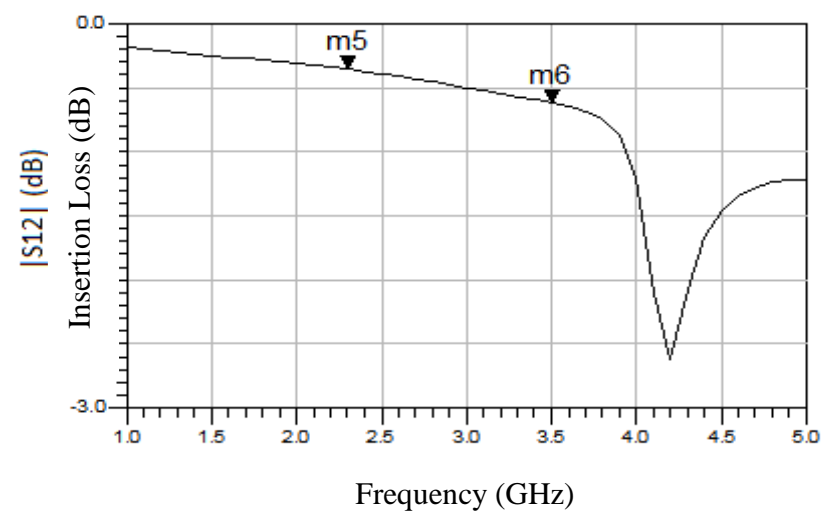

(c)

Figure 10. Performance Result of the Proposed SPDT switch with Open Stub Resonators, (a) Dual Isolation Band, $S_{31}$, (b) Return Loss, $S_{11}$ and (c) Insertion Loss, $S_{21}$

Table 2. Performance Summary of Dual Isolation Band of SPDT switch in $2.3 \mathrm{GHz}$ and $3.5 \mathrm{GHz}$ Bands

\begin{tabular}{cccc}
\hline Frequency Band & Isolation & Return Loss & Insertion Loss \\
\hline $2.3 \mathrm{GHz}$ & $46.78 \mathrm{~dB}$ & $20.83 \mathrm{~dB}$ & $0.359 \mathrm{~dB}$ \\
$3.5 \mathrm{GHz}$ & $44.74 \mathrm{~dB}$ & $15.16 \mathrm{~dB}$ & $0.623 \mathrm{~dB}$ \\
\hline
\end{tabular}


Table 3 lists the SPDT switch designs from other researchers that are the most related to this research work. The isolation performance comparison is made on the same frequency spectrum in 2.5 and 3.5 $\mathrm{GHz}$ bands. It showed that the proposed SPDT switch in this paper is the best in term of high isolation performance with dual isolation band.

Table 3. Comparison of Isolation Performance of SPDT switches from other Researchers

\begin{tabular}{|c|c|c|c|c|c|c|}
\hline & Application & $\begin{array}{c}\text { Design } \\
\text { Approach }\end{array}$ & $\begin{array}{l}\text { Material/ } \\
\text { Package }\end{array}$ & Isolation Technique & $\begin{array}{c}\text { Isolation } \\
\text { at } 2.3 \mathrm{GHz}\end{array}$ & $\begin{array}{c}\text { Isolation } \\
\text { at } 3.5 \mathrm{GHz}\end{array}$ \\
\hline $\begin{array}{l}\text { This } \\
\text { work }\end{array}$ & $\begin{array}{c}\text { Wireless } \\
\text { Communication } \\
\text { System }\end{array}$ & $\begin{array}{l}\text { Dual Band } \\
\text { SPDT }\end{array}$ & $\begin{array}{l}\text { Discrete PIN } \\
\text { diode }\end{array}$ & $\begin{array}{l}\text { Switchable open stub } \\
\text { resonator }\end{array}$ & $46.78 \mathrm{~dB}$ & $44.74 \mathrm{~dB}$ \\
\hline [10] & $\begin{array}{l}\text { WiMAX/ } \\
\text { LTE }\end{array}$ & $\begin{array}{l}\text { Single Band } \\
\text { SPDT }\end{array}$ & $\begin{array}{l}\text { Discrete PIN } \\
\text { diode }\end{array}$ & $\begin{array}{l}\text { Switchable open stub } \\
\text { resonator }\end{array}$ & N.A. & $37 \mathrm{~dB}$ \\
\hline [27] & WiBro & SPST & $\begin{array}{l}\text { Discrete PIN } \\
\text { diode }\end{array}$ & $\begin{array}{l}\text { Switchable dual mode ring } \\
\text { resonator }\end{array}$ & $43.3 \mathrm{~dB}$ & N.A. \\
\hline [28] & WiMAX & SPDT MMIC & FET: pHEMT & Series-Shunt FET & N.A. & $28 \mathrm{~dB}$ \\
\hline [29] & Radar & SPDT & $\begin{array}{l}\text { Discrete PIN } \\
\quad \text { diode }\end{array}$ & $\begin{array}{l}\text { Series PIN diode with } \\
\text { compensation } \\
\text { parasitic capacitance }\end{array}$ & N.A. & $19 \mathrm{~dB}$ \\
\hline [30] & $\begin{array}{c}\text { General } \\
\text { Application }\end{array}$ & SPST & $\begin{array}{c}\text { Discrete PIN } \\
\text { diode }\end{array}$ & Switchable resonator & N.A. & $35 \mathrm{~dB}$ \\
\hline
\end{tabular}

\section{CONCLUSION}

The theory of open stub resonator based on the mathematical model and the realization of the resonator using the microstrip transmission line has been discussed. Analytical results showed that the different values of attenuation pole of transmission line stub resonator for different widths were simply due to the varying values of impedance, $Z_{s}$ of the resonator. By cooperating a commercialized PIN diode in the switchable open stub resonator, it was found that the PIN diode shifted the resonant frequency of the resonator to the lower frequency and also degraded the attenuation level of the bandstop response of the resonator. The open stub stub resonator was implemented in the dual isolation band of SPDT switch circuit where two different open stub resonators were resonated at 2.3 and $3.5 \mathrm{GHz}$ and can be switched between bandstop and allpass responses. The performance results of the proposed SPDT switch showed that the isolation was greater than $30 \mathrm{~dB}$, return loss was greater than $10 \mathrm{~dB}$ and insertion loss less than $2 \mathrm{~dB}$ at the center resonant frequency of 2.3 and $3.5 \mathrm{GHz}$. The potential application of the proposed SPDT switch is for multi band RF front-end system such as WiMAX, LTE, WiFi and HyperLAN.

\section{ACKNOWLEDGEMENTS}

We are grateful to Center for Telecommunication Research and Innovation (CeTRI) and Universiti Teknikal Malaysia Melaka (UTeM) for their encouragement and help for supporting financially to complete this research work.

\section{REFERENCES}

[1] M. R. Hamid, et al., "Vivaldi Antenna With Integrated Switchable Band Pass Resonator," Antennas and Propagation, IEEE Transactions, vol/issue: 59(11), pp. 4008-4015, 2011.

[2] F. Malek, et al., "Complementary Structure of Quadruple P-Spiral Split Ring Resonator ( QPS-SRR ) on Modified Minkowski Patch Antenna Design," Applied Electromagnetics (APACE), 2012 IEEE Asia-Pacific Conference, pp. 142-147, 2012.

[3] G. S. Rajni and A. Marwaha, "Modeling of Split Ring Resonators Loaded Microstrip Line with Different Orientations," International Journal of Electrical and Computer Engineering (IJECE), vol/issue: 5(6), pp. 1363$1371,2015$.

[4] B. A. Adoum and P. W. Wong, "Miniaturized Matched Band-Stop Filter Based Dual Mode Resonator," National Postgraduate Conference (NPC), pp. 7-9, 2011.

[5] I. C. Hunter, et al., "Passive microwave receive filter networks using low-Q resonators," Microwave Magazine, IEEE, pp. 46-53, 2005.

[6] N. A. Shairi, et al., "SPDT Discrete Switch Design using Switchable Radial Stub Resonator for WiMAX and LTE in $3.5 \mathrm{GHz}$ Band," RF and Microwave Conference (RFM), 2013 IEEE International, pp. 1-5, 2013.

[7] M. Hangai, et al., "Millimeter-Wave High-Power MMIC Switch with Multiple FET Resonators," IEICE Transactions on Electronics, vol/issue: E90-C(9), pp. 1695-1701, 2007. 
[8] Z. Wang and C. Park, "Novel Wideband GaN HEMT Power Amplifier Using Microstrip Radial Stub to Suppress Harmonics," Microwave Symposium Digest. 2012 IEEE MTT-S International, pp. 5-7, 2012.

[9] H. Nornikman, et al., "Effect of Spiral Split Ring Resonator (S-SRR) Structure on Truncated Pyramidal Microwave Absorber Design," Antennas and Propagation (ISAP), 2012 International Symposium on, pp. 1188 - 1191, 2012.

[10] N. A. Shairi, et al., "Design of SPDT Switch with Transmission Line Stub Resonator for WiMAX and LTE in 3.5 GHz Band," ARPN Journal of Engineering and Applied Sciences, vol/issue: 11(5), pp. 3198-3202, 2016.

[11] M. M. Mansour, et al., "Realization of Metamaterial Zeroth-Order Resonators Based on MIM Capacitor," International Journal of Nano Devices, Sensors and Systems (IJ-Nano), vol/issue: 2(1), pp. 7-14, 2013.

[12] S. K. M. Khanfar, et al., "Microwave Ring Resonator with a Quarter-Wavelength Coupled-Line Integration," Wireless Technology and Applications (ISWTA), 2012 IEEE Symposium on, pp. 89-91, 2012.

[13] D. M. Pozar, "Microwave Engineering," John Wiley \& Sons, Inc., 2005.

[14] M. Makimoto and S. Yamashita, "Microwave Resonators and Filters for Wireless Communication: Theory, Design and Application," Springer, 2001.

[15] Jack Browne, "RF Front End Is Reconfigurable," Microwave \& RF, pp. 74-75, 2014.

[16] J. E. Mueller, et al., "Requirements for reconfigurable 4G front-ends," 2013 IEEE MTT-S International Microwave Symposium Digest (IMS), pp. 1-4, 2013.

[17] Z. Brito-Brito, et al., "Recent Advances in Reconfigurable Microwave Filters," 2011 SBMO/IEEE MTT-S International Microwave and Optoelectronics Conference (IMOC 2011), pp. 338-346, 2011.

[18] R. Malmqvist, et al., "Multi-Band and Reconfigurable Front-Ends for Flexible and Multi-Functional RF Systems," Asia-Pacific Microwave Conference 2007, pp. 1-4, 2007.

[19] S. Goswami, et al., "A Frequency-Agile RF Frontend Architecture for Multi-Band TDD Applications," IEEE Journal of Solid-State Circuits, vol/issue: 49(10), pp. 2127-2140, 2014.

[20] H. Younesiraad, et al., "Small Multi-Band Rectangular Dielectric Resonator Antennas for Personal Communication Devices," International Journal of Electrical and Computer Engineering (IJECE), vol/issue: 4(1), pp. 1-6, 2014.

[21] E. J. Naglich, et al., "A Tunable Bandpass-to-Bandstop Reconfigurable Filter With Independent Bandwidths and Tunable Response Shape," Microwave Theory and Techniques, IEEE Transactions on, vol/issue: 58(12), pp. 3770 $3779,2010$.

[22] J. X. Chen, et al., "Tunable And Switchable Bandpass Filters Using Slot-Line Resonators," Progress In Electromagnetics Research, vol. 111, pp. 25-41, 2011.

[23] E. J. Naglich, et al., "Switchless Tunable Bandstop-to-All-Pass Reconfigurable Filter," IEEE Transactions on Microwave Theory and Techniques, vol/issue: 60(5), pp. 1258-1265, 2012.

[24] M. K. Zahari, et al., "Reconfigurable Dual-Mode Ring Resonator Matched Bandstop Filter," Wireless Technology and Applications (ISWTA), 2012 IEEE Symposium on, pp. 71-74, 2012.

[25] A. M. S. Zobilah, et al., "RF Switches in Wide- , Broad- , and Multi-Band RF Front-End of Wireless Communications : An Overview," ARPN Journal of Engineering and Applied Sciences, vol/issue: 11(5), pp. 3244 3248, 2016.

[26] P. Y. Chang and Y. S. Lin, "Electronically Switchable Microstrip Bandpass Filter with Good Selectivity," Microwave Conference Proceedings (APMC), 2011 Asia-Pacific, vol. 1, pp. 1158-1161, 2011.

[27] W. S. Lee, et al., "A Band-Stop RF Switch Using a Dual-Mode Stepped-Impedance Microstrip Ring Resonator," Microwave Conference (EuMC), 2010 European, pp. 316-319, 2010.

[28] Y. Hsu, et al., "Single-chip RF Front-end MMIC using InGaAs E/D-pHEMT for 3 . 5 GHz WiMAX Applications," European Microwave Conference 2007, pp. 419-422, 2007.

[29] M. U. Nazir, et al., "PIN Diode Modelling for Simulation and Development of High Power Limiter, Digitally Controlled Phase Shifter and High Isolation SPDT Switch," Proceedings of 2013 10th International Bhurban Conference on Applied Sciences \& Technology (IBCAST), pp. 439-445, 2013.

[30] H. R. Ahn and B. Kim, "A Ring Filter Switch for A Low Loss Wideband and Very Sharp Bandstop Filter," Microwave and Optical Technology Letters, vol/issue: 49(11), pp. 2828-2830, 2007.

\section{BIOGRAPHIES OF AUTHORS}

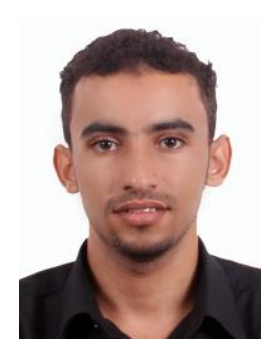

Mohammed Yahya Algumaei was born in IBB, Yemen in 1991. He currently doing his B. Eng. in Electronic Engineering at Faculty of Electronic and Computer Engineering (FKEKK), Universiti Teknikal Malaysia Melaka (UTeM), where his Final Year Project focuses on RF switch-microwave filtering. 


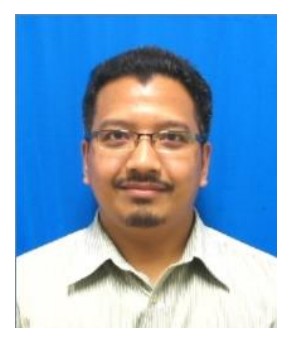

Noor Azwan Shairi, PhD, MIEEE - was born in Malaysia in 1978. He received the Bachelor in Engineering (Electrical-Telecommunication) and the Master in Electrical Engineering (By Research) from Universiti Teknologi Malaysia (UTM), in 2002 and 2005, respectively. In 2015, he obtained his Doctorate from Universiti Teknikal Malaysia Melaka (UTeM) in the field of Electronic Engineering (RF and Microwave). Currently, he is a Lecturer at the Faculty of Electronics and Computer Engineering (FKEKK), Universiti Teknikal Malaysia Melaka (UTeM), Malaysia. His research areas are mainly focused on RF and Microwave Engineering.

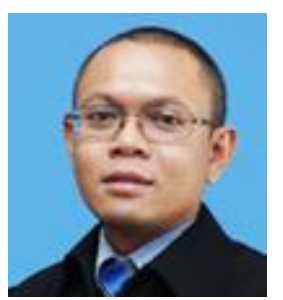

Zahriladha Zakaria, PhD, MIEEE - was born in Malaysia in 1975. He received the B. Eng. and M. Eng. in Electrical and Electronic Engineering from the Universiti Teknologi Malaysia in 1998 and 2004 respectively. He obtained his Doctorate from The University of Leeds in 2010 in the field of Microwave Engineering. He is currently an Associate Professor at the Faculty of Electronic and Computer Engineering, Universiti Teknikal Malaysia Melaka (UTeM), Malaysia. His research interests include a variety of microwave device developments such as planar and nonplanar microwave filters, amplifiers and antennas.

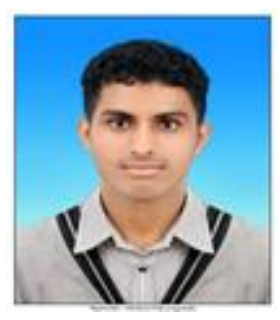

Abdullah Zobilah was born in AL-Hudaida, Yemen in 1990. He received his B. Eng. degree in Wireless Communication Engineering from Universiti Teknikal Malaysia Melaka in 2015. Currently, He is a master student at the same University, Faculty of Electronic and Computer Engineering (FKEKK), where his master research focuses on RF switches.

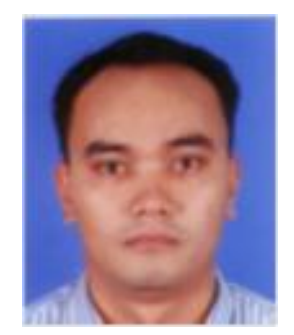

Badrul Hisham Ahmad graduated with honours degree in Electronic and Communication Engineering from the University of Leeds, UK in 1998. He received the M.Sc. degree from University Kebangsaan Malaysia in 2003 and Ph.D. degree from Leeds University, United Kingdom for a thesis on the design and development of SIW filters in 2008. He is a lecturer at Universiti Teknikal Malaysia Melaka since 2003. He is also an active researcher with a good track record with a number of research projects in RF and Microwave Engineering. 